\title{
Diffuse malignant mesothelioma of the peritoneum and pleura, analysis of markers
}

\author{
Jacqueline K Trupiano${ }^{1}$, Kim R Geisinger ${ }^{1}$, Mark C Willingham ${ }^{1}$, Paul Manders ${ }^{1}$, \\ Nora Zbieranski ${ }^{1}$, Doug Case $^{2}$ and Edward A Levine ${ }^{3}$ \\ ${ }^{1}$ Department of Pathology; ${ }^{2}$ Department of Public Health Sciences and ${ }^{3}$ Department of Surgery, Wake Forest \\ University School of Medicine, Winston-Salem, NC, USA
}

\begin{abstract}
Diffuse malignant mesothelioma of the peritoneum is a rare diagnosis. Despite many histopathologic similarities between peritoneal and pleural tumors, clinical and prognostic features may be quite different. There is a paucity of data evaluating molecular features of peritoneal mesotheliomas. Therefore, we compared the results of a battery of immunohistochemical markers, some with therapeutic implications, in patients with primary peritoneal or pleural mesotheliomas. We examined 24 peritoneal and nine pleural malignant mesotheliomas with a battery of immunohistochemical markers (cytokeratin AE1/3, calretinin, c-kit/CD117, desmin, epidermal growth factor receptor (EGFR), estrogen receptors (ER), progesterone receptors (PR), MIB-1, and cleaved caspase-3) in an attempt to distinguish any differences in this tumor arising in these two distinct locations. The results indicate that the only marker to show a significant difference in its staining pattern between these two sites was EGFR $(P=0.0004)$. In all, 92\% $(22 / 24)$ of peritoneal tumors demonstrated $3+$ or $4+$ immunoreactivity with EGFR, opposed to only $33 \%(3 / 9)$ pleural tumors. There was no significant difference in immunoreactivity between the pleural and peritoneal tumors with c-kit, ER, PR, cleaved caspase 3, calretinin, and desmin. There was a trend toward increased cytokeratin $(P=0.07)$ and MIB-1 $(P=0.08)$ expression in the peritoneal group. There was no significant difference in age, sex, or histologic subtype between the two locations. In conclusion, despite similarities between peritoneal and pleural mesothelioma, there are differences between this neoplasm arising in these two sites. The EGFR expression is more pronounced in peritoneal tumors compared to pleural tumors. The increased expression of EGFR in the peritoneal lesions may be of clinical significance with the recent emergence of epidermal growth factor receptor-targeted therapies. Modern Pathology (2004) 17, 476-481, advance online publication, 20 February 2004; doi:10.1038/modpathol.3800067
\end{abstract}

Keywords: malignant mesothelioma; peritoneum; pleural; immunohistochemistry

Malignant mesothelioma is a rare tumor with an annual incidence in the United States of approximately 2500 cases per year. ${ }^{1}$ Pleural malignant mesotheliomas are 10-30-fold more common than their peritoneal counterparts. ${ }^{2,3}$ Regardless of site of origin, the prognosis is usually poor with a median survival of 4-12 months for pleural tumors ${ }^{4}$ and less than 1 year for peritoneal tumors. ${ }^{3}$ Systemic chemotherapy and radiation does little to improve the outcome. Since diaphragmatic extension is common to both pleural and peritoneal mesotheliomas, it is not uncommon to have secondary pleural invasion from peritoneal disease, or vice versa, making the distinction of peritoneal from pleural tumors difficult clinically. In an attempt to better clarify the

Correspondence: E Levine, Department of General Surgery, Baptist Medical Center, Medical Center Boulevard, WinstonSalem, NC 27157, USA.

E-mail: elevine@wfubmc.edu

Received 30 October 2003; revised 30 December 2003; accepted 31 December 2003; published online 20 February 2004 surface of origin for mesothelioma, and identify any possible molecular targets for therapy and immunohistochemical differences between tumors arising in these two sites, we embarked on this comparison of pleural and peritoneal mesotheliomas.

We examined 24 peritoneal and nine pleural malignant mesotheliomas with a host of markers (cytokeratin AE1/3, calretinin, CD117, desmin, EGFR, ER, PR), including a proliferative marker (MIB-1) and a marker of apoptotic cell death (cleaved caspase-3), attempting to elucidate any differences between tumors arising in these two distinct locations.

\section{Materials and methods}

Malignant mesotheliomas accessioned between 1990 and 2003 within the Department of Pathology of Wake Forest University Baptist Medical Center were retrieved. Cases were accepted as mesothelioma if the light microscopy, immunohistochemistry, 
and clinical/surgical findings were fully consistent with the diagnosis. Benign mesothelial lesions, such as adenomatoid tumor, well-differentiated papillary mesothelioma, localized fibrous tumors, and multicystic mesothelioma were excluded from the study. Tissue for light microscopy was routinely fixed in $10 \%$ neutral-buffered formalin and processed for paraffin embedding and stained with hematoxylin and eosin. Most of the mesotheliomas were classified into one of the three characteristic histologic subtypes: epithelial, sarcomatoid (fibrous), and biphasic. One desmoplastic and one deciduoid variant were also in the cohort.

Immunohistochemistry was performed with the labeled streptavidin-biotin system by means of an automated immunostainer (Ventana NexES; Ventana Medical Systems, Tucson, AZ, USA). The following monoclonal antibodies were applied: AE1/3 (1:200; DAKO, Carpinteria, CA, USA), calretinin (prediluted; Ventana, Tucson, AZ, USA), CD117 (1:400; DAKO, Carpinteria, CA, USA), ER (prediluted; Ventana, Tucson, AZ, USA), PR (prediluted; Ventana, Tucson, AZ, USA), EGFR (1:20; Zymed, San Fransisco, CA, USA), desmin (1:50 with no pretreatment, DAKO, Carpinteria, CA, USA), MIB-1/Ki-67 (1:80, DAKO, Carpinteria, CA, USA), and cleaved caspase-3 (1:100, Cell Signaling Technologies, Beverly, MA, USA). Optimal dilutions were determined by running several dilutions of antibody using various pretreatments and then choosing the best dilution based on the intensity of staining vs background. Negative controls consisted of antibody diluent (Cat. \#251-018, Ventana, Tucson, AZ, USA). Positive controls reacted accordingly.

A negative $-4+$ scoring system was used in the interpretation of AE1/3, calretinin, CD117, EGFR, and desmin. Tumors lacking immunoreactivity were classified as negative. Tumors demonstrating immunoreactivity in 1-25\% of their cells were scored as $1+, 26-50 \%$ of cells: $2+, 51-75 \%$ of cells: $3+$, and $76-100 \%$ of cells: $4+$. Estrogen receptor, progesterone receptor, and MIB-1 positivity were reported as a straight percentage of neoplastic cells. As staining may be focal, numerous groups of 20 cells were counted in various fields of the slides and a percentage of positive cells was obtained from the total number of cells counted. Nuclear staining was necessary for ER, PR, and MIB-1 positivity. For cleaved caspase-3 staining, 50 cells were counted and the number of cells demonstrating immunoreactivity, with or without nuclear changes such as pyknosis or karyorrhexis, were counted.

Statistical analysis was performed to determine if there was a significant difference in the immunohistochemical staining results between the pleural and peritoneal tumors. Fisher's exact tests were used to assess differences in the categorical characteristics and markers and Wilcoxon rank-sum tests were used to assess differences in the ordinal and continuous variables.

\section{Results}

A total of 33 malignant mesotheliomas were examined, including 24 primary peritoneal and nine primary pleural tumors (Table 1). Patient characteristics were similar for the two groups. The peritoneal cohort consisted of 14 men and 10 women. The age range for the peritoneal group was 23-85 years with a median age of 60 years. In all, 20 (83\%) tumors were classified as epithelial (Figure 1), one $(4 \%)$ as sarcomatoid, one $(4 \%)$ as deciduoid, and two $(8 \%)$ as biphasic. The pleural group consisted of six men and three women. The age range was $48-78$ years with a median age of 66 years. Seven tumors

Table 1 Patient and tumor characteristics for peritoneal and pleural mesotheliomas

\begin{tabular}{|c|c|c|c|}
\hline Characteristic & Peritoneal \# (\%) & Pleural \# (\%) & $P$-value \\
\hline Total & $24(100)$ & $9(100)$ & \\
\hline Age-median (range) & $60.5(23-85)$ & $66.0(48-78)$ & 0.5046 \\
\hline \multicolumn{4}{|l|}{ Gender } \\
\hline Female & $10(42)$ & $3(33)$ & 1.0 \\
\hline Male & $14(58)$ & $6(67)$ & \\
\hline Histology & $20(83)$ & $7(78)$ & 0.4410 \\
\hline Epithelial & $2(8)$ & $0(0)$ & \\
\hline Biphasic & $1(4)$ & $1(11)$ & \\
\hline Sarcomatoid & $1(4)$ & $0(0)$ & \\
\hline Deciduoid & $0(0)$ & $1(11)$ & \\
\hline \multicolumn{4}{|l|}{ Desmoplastic } \\
\hline$C-K I T$ & & & - $^{\mathrm{a}}$ \\
\hline Negative & $24(100)$ & $9(100)$ & \\
\hline \multicolumn{3}{|l|}{$E R$} & 1.0 \\
\hline Negative & $22(92)$ & $9(100)$ & \\
\hline Positive & $2(8)$ & $0(0)$ & \\
\hline \multicolumn{3}{|l|}{$P R$} & $-^{\mathrm{a}}$ \\
\hline Negative & $24(100)$ & $9(100)$ & \\
\hline \multicolumn{4}{|l|}{ EGFR } \\
\hline 0 & $1(4)$ & $1(11)$ & 0.0004 \\
\hline $1+$ & $1(4)$ & $2(22)$ & \\
\hline $2+$ & $0(0)$ & $3(33)$ & \\
\hline $3+$ & $3(12)$ & $2(22)$ & \\
\hline $4+$ & $19(79)$ & $1(11)$ & \\
\hline \multicolumn{4}{|l|}{ MIB-1 } \\
\hline Negative & $6(25)$ & $3(33)$ & 0.0824 \\
\hline Positive & $18(75)$ & $6(67)$ & \\
\hline Median (range) & $0.03(0-0.15)$ & $0.01(0-0.05)$ & \\
\hline \multicolumn{4}{|l|}{ Caspase-3 } \\
\hline 0 & $19(79)$ & $7(78)$ & 1.0 \\
\hline 1 & $2(8)$ & $1(11)$ & \\
\hline 2 & $1(4)$ & $1(11)$ & \\
\hline 3 & $2(8)$ & $0(0)$ & \\
\hline \multicolumn{3}{|l|}{ Desmin } & $-^{a}$ \\
\hline Negative & $24(100)$ & $9(100)$ & \\
\hline \multicolumn{4}{|l|}{ Cytokeratin } \\
\hline $1+$ & $0(0)$ & $1(11)$ & 0.0696 \\
\hline $2+$ & $1(4)$ & $1(11)$ & \\
\hline $3+$ & $1(4)$ & $1(11)$ & \\
\hline \multirow{2}{*}{\multicolumn{4}{|c|}{$\begin{array}{l}4+ \\
\text { Calretinin }\end{array}$}} \\
\hline & & & \\
\hline 0 & $4(17)$ & $2(22)$ & 0.2226 \\
\hline $1+$ & $4(17)$ & $2(22)$ & \\
\hline $2+$ & $0(0)$ & $0(0)$ & \\
\hline $3+$ & $6(25)$ & $4(44)$ & \\
\hline $4+$ & $10(42)$ & $1(11)$ & \\
\hline
\end{tabular}

${ }^{\mathrm{a}}$ No statistics calculated since all subjects were negative in both groups. 


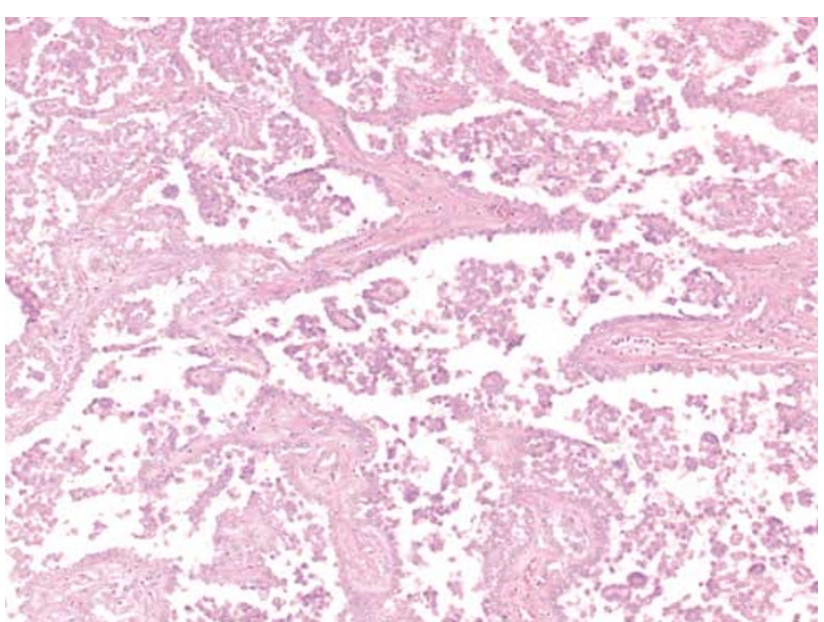

Figure 1 Malignant epithelial mesothelioma (hematoxylin \& eosin stain; original magnification $\times 200$ ).

$(78 \%)$ were classified as epithelial, one $(11 \%)$ sarcomatoid, and one (11\%) as desmoplastic. There was no significant difference in age $(P=0.50)$, gender $(P=1.0)$, or histologic subtype $(P=0.44)$ between mesotheliomas arising in either location.

The results of immunohistochemical staining for peritoneal and pleural tumors are also summarized in Table 1. All tumors, regardless of site, were found to be negative for c-kit (CD117), desmin, and PR. All tumors demonstrated some degree of immunoreactivity for cytokeratin AE1/3, but the peritoneal tumors tended to be more diffusely positive $(P=0.07)$. Calretinin was positive in 20 of 24 $(83 \%)$ peritoneal tumors and seven or nine $(78 \%)$ pleural tumors $(P=1.0)$. Estrogen receptor positivity was demonstrated in two peritoneal tumors and zero pleural tumors $(P=1.0)$. Both ER-positive tumors occurred in women and one was of the deciduoid subtype.

Of $24,18(79 \%)$ peritoneal tumors and six of nine $(67 \%)$ pleural tumors demonstrated some degree of immunoreactivity for MIB-1. In positive cases, the percentage of MIB-1 positive cells varied between 1$15 \%$ in peritoneal tumors and $1-5 \%$ in pleural tumors. The difference in MIB-1 distributions was of borderline statistical significance $(P=0.08)$.

Positive staining for caspase-3 was identified in five of $24(21 \%)$ peritoneal and two of nine $(22 \%)$ pleural tumors. The percentage of positive cells was similar in both groups varying from 1 to $3 \%$ in the peritoneal group and 1 to $2 \%$ in the pleural group. The distributions of caspase- 3 were not found to be significantly different between the two groups $(P=0.95)$.

The examined marker that demonstrated the greatest difference in its staining pattern between the pleural and peritoneal-based tumors was EGFR $(P=0.0004)$. Of all mesotheliomas examined from both sites, 31 of 33 tumors (94\%) demonstrated immunoreactivity for EGFR (Figure 2). Of the two

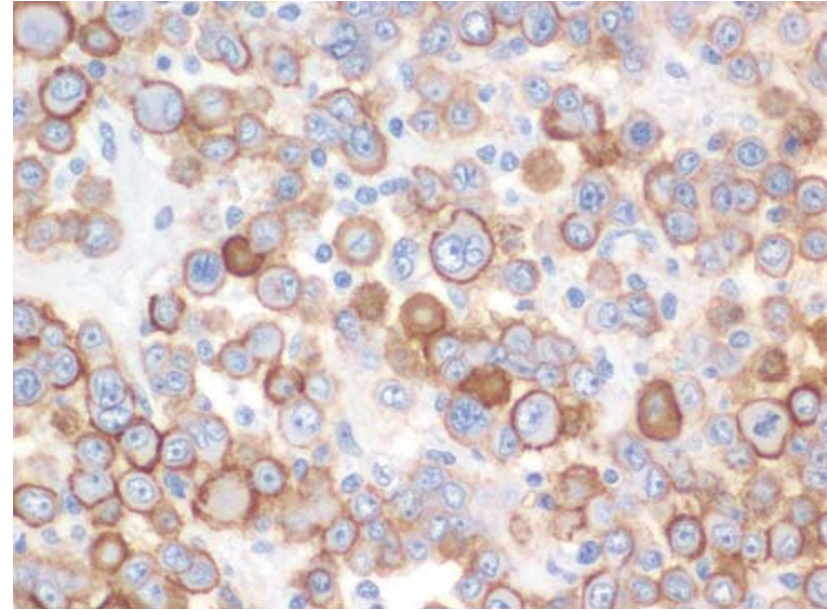

Figure 2 Malignant mesothelioma demonstrating strong, membranous immunoreactivity for EGFR (immunohistochemical stain for EGFR; original magnification $\times 400$ ).

tumors that were negative, one was an epithelial peritoneal tumor and one a sarcomatoid pleural tumor. Of the remaining 23, peritoneal tumors 22 showed $3+$ to $4+$ reactivity, whereas only three of nine $(33 \%)$ pleural tumors were as diffusely positive. In all, $56 \%$ of pleural tumors (5/9) showed only $1+$ to $2+$ reactivity.

\section{Discussion}

Malignant mesothelioma is a neoplasm originating from the mesothelial surface lining cells of the serous body cavities. Tumors typically originate from the pleura or, less commonly, the peritoneum and rarely, the pericardium and tunica vaginalis testis. ${ }^{3}$ Malignant peritoneal mesothelioma (MPM) is a rare malignancy characterized by primary neoplastic change involving the lining surfaces of the peritoneal cavity. Widespread peritoneal dissemination is typical at presentation clinically manifesting as abdominal discomfort/distention, digestive disturbances, weight loss, and symptomatic malignant ascites. Cytologic analysis of fluid in this setting may be helpful, but has a low yield, with most cases being diagnosed with laparotomy or laparoscopy.

MPM is much less common than its pleural counterpart, accounting for approximately $10 \%$ of all malignant mesotheliomas. ${ }^{1,2,5}$ The median age at diagnosis in the literature and our experience is 60 , and there is a male:female ratio of $3: 1^{1}$ The majority of patients have a history of asbestos exposure, although this association is much less common in females ${ }^{1,6,7}$ Diffuse peritoneal mesothelioma is often described at surgery as numerous small (1-5 mm), shiny, whitish nodules carpeting all visualized peritoneal surfaces. Many larger $(2-5 \mathrm{~cm})$ nodular excrescences and infiltrative masses are also often present. The tumor almost always infiltrates the entire omentum and, in advanced stages, 
involvement of the pleural cavity through transdiaphragmatic extension and distant metastatic disease may be seen. ${ }^{1,3,6}$

The histologic features of MPM are generally identical to their pleural counterparts and divided into epithelial, sarcomatoid, and biphasic subtypes. Other, less common, subtypes have also been described. The distribution of the various histologic subtypes varies from series to series, but epithelial tumors predominate in both pleural and peritoneal locations. In the series by Kannerstein and Churg ${ }^{8}$ which examined 82 peritoneal tumors, $75.6 \%$ were epithelial, $22 \%$ biphasic, and $2.4 \%$ sarcomatoid. Sarcomatoid tumors are far more common in the pleura than the peritoneal cavity. This was not demonstrated in our data, although our cohort was admittedly small. Rare peritoneal tumors are characterized by a sheet-like pattern of polygonal cells with abundant eosinophilic cytoplasm, distinct cell membranes, and low nuclear to cytoplasmic ratios, the so-called deciduoid variant. This variant typically affects the peritoneum of young women and pursues an aggressive, often fatal, clinical course. ${ }^{9}$ Ordonez ${ }^{10}$ recently reported four cases of pleural deciduoid mesothelioma, with three of the four cases arising in males, two of which gave a history of asbestos exposure. ${ }^{10}$ One deciduoid tumor was identified in this study and involved the peritoneum of a 27-year-old female.

The prognosis for mesothelioma is poor. Untreated, survival ranges from 9 to 18 months for peritoneal tumors and 4 to 12 months for pleural tumors. ${ }^{1,4,11}$ Treatment modalities do little to prolong survival. Complete surgical resection is usually not feasible. Radiation therapy cannot be given in sufficient doses to eradicate disease, and systemic chemotherapeutic agents are notoriously ineffective in most patients. ${ }^{1}$ However, recent trials of multimodality therapy, including cytoreductive surgery with intraperitoneal chemotherapy, have resulted in improved survival in many patients. ${ }^{1,4,12-16}$

Immunohistochemistry is an important ancillary technique in the diagnosis of mesothelioma, particularly in differentiating mesotheliomas from adenocarcinoma and sarcoma. This distinction is exceedingly important for both therapeutic and medico-legal purposes. Mesotheliomas demonstrate a common immunohistochemical profile, regardless of the site of origin. A host of glycoproteins, including carcinoembryonic antigen, BerEp4, LeuM1, and B72.3, have been shown to be preferentially expressed by adenocarcinomas, with absent or limited expression in mesotheliomas. ${ }^{19,20}$ Thus, the diagnosis of mesothelioma historically rested largely on the absence of immunoreactivity for these markers. ${ }^{17-20}$ Antigens more commonly expressed in mesotheliomas than carcinomas have recently been described and include calretinin, cytokeratin 5/6, HBME-1, N-cadherin, and thrombomodulin. ${ }^{17-21}$ Thus, histopathology coupled with an immunohistochemical panel aids in accurate diagnosis.
In the current study, we examined a panel of immunohistochemical markers in an attempt to identify potential therapeutic targets and any differences in the staining characteristics of peritoneal versus pleural mesotheliomas. All tumors demonstrated immunoreactivity for cytokeratin AE1/3. Calretinin was positive in 20 of $24(83 \%)$ peritoneal tumors and seven or nine (78\%) pleural tumors. All tumors, regardless of site, were found to be negative for c-kit (CD117), desmin, and PR. Horvai et al. ${ }^{22}$ recently examined c-kit expression in 37 mesotheliomas. Although a subset of cases showed nuclear immunoreactivity, all cases were negative for c-kit mRNA by RT-PCR. Estrogen receptor positivity was demonstrated in two peritoneal tumors. Both ERpositive tumors occurred in women and one was of the deciduoid subtype. These findings suggest that ST1-517 (Gleevec) and selective receptor modifiers and aromatase inhibitors are unlikely to be of therapeutic value.

MIB-1 (Ki-67) is a nuclear antigen expressed in greatest concentration during specific parts of the cell cycle and there is a good correlation between MIB-1 expression and degree of cell proliferation. ${ }^{23}$ In all, 18 of $24(75 \%)$ peritoneal tumors and six of nine $(67 \%)$ pleural tumors demonstrated some degree of immunoreactivity for MIB-1. In positive cases, the percentage of MIB-1 positive cells varied between $1-15 \%$ in peritoneal tumors and $1-5 \%$ of pleural tumors. While this difference was of borderline statistical significance $(P=0.08)$, this cohort is admittedly small to draw any significant conclusions.

The caspase family of serine proteases plays an essential role in the initiation and regulation of the proteolytic events occurring during apoptosis. ${ }^{24,25}$ Caspase-3 is one of the primary executioners of apoptosis, necessary for the cleavage of a large number of proteins and for apoptosis-associated chromatin margination, DNA fragmentation, and nuclear collapse during apoptosis. ${ }^{26}$ The detection of activated caspase-3 could, therefore, be a valuable and specific tool for identifying apoptotic cells in tissue sections, before the morphologic features of apoptosis are evident. ${ }^{24,25}$ Positive staining was identified in five of $24(21 \%)$ of peritoneal and two of nine $(22 \%)$ of pleural tumors. The percentage of positive cells was similar in both groups varying from 1 to $3 \%$ in the peritoneal group and 1 to $2 \%$ in the pleural group.

The examined marker that demonstrated the most statistically significant difference in its staining pattern between the pleural and peritonealbased tumors was EGFR, which demonstrated much greater positivity in the peritoneal tumors. In all, $91 \%$ of peritoneal tumors showed $3+-4+$ staining, whereas only $33 \%$ of pleural tumors stained as diffusely $(P=0.0004)$. Epidermal growth factor receptor binds epidermal growth factor (EGF), which has tyrosine kinase activity and is mitogenic for a variety of epithelial cells and fibroblasts 
in vitro. Epidermal growth factor receptor activity is an area of intense research in many malignancies due to the recent development of many therapeutic agents targeted against the activity of this receptor. Several human cancers, including cancers of the upper aerodigestive tract (non-small-cell carcinoma of the lung, head and neck, esophagus, and stomach), colon, pancreas, breast, ovary, bladder, and kidney have been shown to display EGFR RNA and/or overexpression of the protein. ${ }^{26}$ In one study, EGFR expression was demonstrated by immunohistochemistry in $68 \%$ of paraffin-embedded MPM specimens. ${ }^{27}$ Many therapeutic agents have recently been developed to abrogate this receptor's activity. Janne et al. demonstrated that inhibition of EGFR signaling by ZD1839 (Iressa) led to marked antiproliferative effects of all MPM cell types examined in vitro. ${ }^{28}$ Many studies have also demonstrated a potentiation of the antitumor effects of cytotoxic chemotherapy agents by Iressa in soft agarose and in xenograft models. ${ }^{28}$ Both chemotherapy and radiation are used in the treatment of MPM, and Iressa treatment may enhance the effects of these modalities. In vitro studies in mesothelioma combining radiation and chemotherapy with Iressa are presently underway. ${ }^{13,28}$

Malignant mesotheliomas of the peritoneum or pleura are rare tumors portending a poor prognosis. Many clinical, laboratory, and histologic parameters have been investigated in an attempt to better characterize this group of rare neoplasms. Many immunohistochemical markers were evaluated in the current study assessing for differences between the peritoneal and pleural groups. Epidermal growth factor receptor expression was significantly higher in the peritoneal group compared to the pleural group. The clinical, biologic, prognostic, and therapeutic implications of this, if any, are unclear. Whether patients with malignant mesotheliomas may benefit or not from EGFR-targeted therapies remains to be determined.

\section{References}

1 Taub RN, Keohan ML, Chabot JC, et al. Peritoneal mesothelioma. Curr Treat Options Oncol 2000;1: 303-312.

2 Loggie BW. Malignant peritoneal mesothelioma. Curr Treat Options Oncol. 2001;2:395-399.

3 Sebbag G, Yan H, Shmookler BM, et al. Results of treatment of 33 patients with peritoneal mesothelioma. Br J Surg 2000;87:1587-1593.

4 Zellos LS, Sugarbaker DJ. Diffuse malignant mesothelioma of the pleural space and its management. Oncology 2002;16:907-925.

5 Kerrigan SA, Turnnir RT, Clement PB, et al. Diffuse malignant epithelial mesotheliomas of the peritoneum in women: a clinicopathologic study of 25 patients. Cancer 2002;94:378-385.

6 Goldblum J, Hart WR. Localized and diffuse mesotheliomas of the genital tract and peritoneum in women. A clinicopathologic study of nineteen true mesothelial neoplasms, other than adenomatoid tumors, multicystic mesotheliomas, and localized fibrous tumors. Am J Surg Pathol 1995;19:1124-1137.

7 Asensio JA, Goldblatt P, Thomford NR. Primary malignant peritoneal mesothelioma. A report of seven cases and a review of the literature. Arch Surg 1990;125:1477-1481.

8 Kannerstein M, Churg J. Peritoneal mesothelioma. Hum Pathol 1977;8:83-94.

9 Nascimento AG, Keeney GL, Fletcher CDM. Deciduoid peritoneal mesothelioma. An unusual phenotype affecting young females. Am J Surg Pathol 1994;18: 439-445.

10 Ordonez NG. Epithelial mesothelioma with deciduoid features. Am J Surg Pathol 2000;24:816-823.

11 van Ruth S, Baas P, Zoetmulder FAN. Surgical treatment of malignant pleural mesothelioma, a review. Chest 2003;123:551-561.

12 Loggie BW, Fleming RA, McQuellon RP, et al. Prospective trial for the treatment of malignant peritoneal mesothelioma. Am J Surg 2001;67: 999-1003.

13 Nowak AK, Lake RA, Kindler HL, et al. New approaches for mesothelioma: biologics, vaccines, gene therapy, and other novel agents. Semin Oncol 2002;29:82-96.

14 de Bree E, van Ruth S, Baas P, et al. Cytoreductive surgery and intraoperative hyperthermic intrathoracic chemotherapy in patients with malignant pleural mesothelioma or pleural metastases of thymoma. Chest 2002;121:480-487.

15 Girling DJ, Muers MF, Qian W, et al. Multicentric randomized controlled trial of the management of unresectable malignant mesothelioma proposed by the british thoracic society and the british medical research council. Semin Oncol 2002;29: 97-101.

16 Park BJ, Alexander HR, Libutti SK, et al. Treatment of primary peritoneal mesothelioma by continuous hyperthermic peritoneal perfusion (CHPP). Ann Surg Oncol 1999;6:582-590.

17 Riera JR, Astento-Osuna CA, Longmate JA, et al. The immunohistochemical diagnostic panel for epithelial mesothelioma. A reevaluation after heat-induced epitope retrieval. Am J Surg Pathol 1997;21:1409-1419.

18 Ordonez NG. Role of immunohistochemistry in distinguishing epithelial peritoneal mesotheliomas from peritoneal and ovarian serous carcinomas. Am J Surg Pathol 1998;22:1203-1214.

19 Ordonez NG. The immunohistochemical diagnosis of epithelial mesothelioma. Hum Pathol 1999;30: 313-323.

20 Ordonez NG. Value of cytokeratin 5/6 immunostaining in distinguishing epithelial mesothelioma of the pleura from lung adenocarcinoma. Am J Surg Pathol 1998;22:1215-1221.

21 Attanoos RL, Webb R, Dojcinov SD, et al. Value of mesothelial and epithelial antibodies in distinguishing diffuse peritoneal mesothelioma in females from serous papillary carcinoma of the ovary and peritoneum. Histopathology 2002;40:237-244.

22 Horvai AE, Li L, Zhidong X, et al. C-Kit is not expressed in malignant mesothelioma. Mod Pathol 2003;16:818-822.

23 Beer TW, Buchanan R, Matthews AW, et al. Prognosis in malignant mesothelioma related to MIB 1 prolifera- 
tion index and histologic subtype. Hum Pathol 1998;29:246-251.

24 Duan WR, Garner DS, Williams SD, et al. Comparison of immunohistochemistry for activated caspase-3 and cleaved cytokeratin 18 with the TUNEL method for quantification of apoptosis in histological sections of PC-3 subcutaneous xenografts. J Pathol 2003;199: 221-228.

25 Omezzine A, Chater S, Mauduit C, et al. Long-term apoptotic cell death process with increased expression and activation of caspase- 3 and -6 in adult rat germ cells exposed in utero to flutamide. Endocrinology 2003;144:648-661.
26 Arteaga CL, Baselga J. Clinical trial design and end points for epidermal growth factor receptor-targeted therapies: implications for drug development and practice. Clin Cancer Res 2003;9:1579-1589.

27 Dazzi H, Hasleton P, Thatcher N, et al. Malignant pleural mesothelioma and epidermal growth factor receptor (EGF-R). Relationship of EGF-R with histology and survival using fixed paraffin-embedded tissue and the F4 monoclonal antibody. Br J Cancer 1990;61:924-926.

28 Janne PA, Taffaro ML, Salgia R, et al. Inhibition of epidermal growth factor receptor signaling in malignant pleural mesothelioma. Cancer Res 2002;62: 5242-5247. 